\title{
Localized vitreous adhesion to the retina after ocular contusion with a baseball
}

This article was published in the following Dove Press journal:

Clinical Ophthalmology

7 June 2012

Number of times this article has been viewed

\section{Masayo Kimura}

Akira Nishimura

Kazuhisa Sugiyama

Department of Ophthalmology and Visual Science, Kanazawa University Graduate School of Medical Science, Kanazawa, Japan
Correspondence: Akira Nishimura Department of Ophthalmology and Visual Science, Kanazawa University Graduate School of Medical Science, I3-I Takara-machi, Kanazawa 920-8641, Japan Tel +8I 762652403

Fax +8I 762229660

Email eyenishi@med.kanazawa-u.ac.jp
Purpose: To report a series of five cases of vitreous adhesions after blunt trauma caused by a baseball strike.

Methods: The medical records of patients with ocular contusion after being struck by a baseball, who had undergone pars plana vitrectomy, were reviewed. An aqueous suspension of triamcinolone acetonide was used intraoperatively to facilitate visualization of the vitreous.

Results: Five eyes were reviewed in this study. There were two cases of retinal detachment, two cases of traumatic macular hole, and one case of retinal detachment due to traumatic macular hole. Despite the surgical creation of posterior vitreous detachment, if not already present, the thin layer of localized vitreous adhesion to the retinal necrotic area with/without retinal hole was found between the major vascular arcades and the equator in all cases. The thin layer of the vitreous was removed with a vitreous cutter and diamond-dusted membrane scraper, but complete removal was impossible.

Conclusions: A thin layer of localized vitreous adhesion at the area of retinal degeneration was observed in eyes following ocular contusion.

Keywords: localized, vitreous adhesion, triamcinolone acetonide, ocular contusion, pars plana vitrectomy

\section{Introduction}

Non-penetrating ocular contusion, or blunt ocular trauma, leads to various retinal changes, such as commotio retinae, traumatic macular hole $(\mathrm{MH})$, contusion retinal necrosis, and retinal breaks, including retinal detachment (RD). Commotio retinae is reported to be the result of swelling of the axons of photoreceptors, Müller cell processes, and the inner and outer segments of photoreceptors. ${ }^{1}$ With severe blunt trauma, several histological changes may be found, including the disruption of photoreceptors and the retinal pigment epithelium, as well as the breakdown of the outer blood-retinal barrier. ${ }^{2}$ Fundus imaging may show relatively common manifestations, such as finely stippled pigmentary alterations ${ }^{3,4}$ or retinitis pigmentosa-like picture..$^{5-7}$ However, there are not many reports on how the vitreous behaves after the impact of an ocular contusion. ${ }^{8-10}$

Some reports have described the residual vitreous cortex in diabetic retinopathy ${ }^{11-14}$ and highly myopic eyes. ${ }^{15-17}$ Following ocular contusion, we observed a thin layer of localized vitreous adhesion at the area of retinal degeneration, even after surgical posterior vitreous detachment (PVD), using triamcinolone acetonide (TA) in order to facilitate the visualization of the vitreous and posterior hyaloids. ${ }^{11-18}$ 


\section{Subjects and methods}

The medical records of patients with blunt ocular trauma after being struck by a baseball, who had undergone pars plana vitrectomy (PPV) between October 2006 and August 2010, were retrospectively reviewed. One patient with a globe rupture secondary to blunt trauma was excluded. Patients were fully informed of all aspects of the procedure, and written informed consent was obtained from each one, in accordance with our institutional guidelines. Institutional review board/ethics committee approval was obtained, and this study followed the tenets of the Declaration of Helsinki.

A consecutive series of five eyes with blunt ocular trauma caused by baseball strike was enrolled. All patients underwent comprehensive ophthalmic examinations at the pre- and postoperative visits, including the measurement of best corrected visual acuity, using the Landolt $\mathrm{C}$ acuity chart, non-contact tonometry, slit-lamp examination of the anterior and posterior segments, and fundus examinations.

All procedures were performed under either general anesthesia or sub-Tenon's anesthesia. Phacoemulsification and intraocular lens implantation were performed on all the patients prior to PPV. The combined cataract surgeries were performed in order to trim the vitreous base as meticulously and completely as possible and to avoid postoperative cataract due to the use of gas or silicone oil (SO) (Silikon 1000; Alcon, Fort Worth, TX) as intraocular tamponade agents, even though all patients in our study were young. All patients underwent a 20- or 23-gauge, 3-port PPV, using a 23- or 25-gauge chandelier light (Synergetics Inc, St Charles, MO). An aqueous suspension of TA (Kenacort-A; 40 mg/mL, Bristol-Myers Squibb $\mathrm{SL}$, Tokyo, Japan), prepared as described previously, ${ }^{19}$ was used intraoperatively to facilitate visualization of the vitreous and posterior hyaloids. ${ }^{11-18}$ In the MH cases, the internal limiting membrane (ILM) around the MH within the major temporal vascular arcades was peeled off with vitreous forceps after the visualization of ILM with TA or indocyanine green (ICG) staining procedure. The ICG solution was prepared by dissolving $25 \mathrm{mg} / \mathrm{per}$ vial of dry ICG dye in $1 \mathrm{~mL}$ of distilled water, and then mixing with $19 \mathrm{~mL}$ of intraocular irrigation solution (BSS plus; Alcon Surgical), to obtain a final ICG concentration of $1.25 \mathrm{mg} / \mathrm{mL}$ $(0.125 \%)$. After central and peripheral vitreous removal, all eyes underwent $360^{\circ}$ scleral depression to trim the vitreous base with the use of a 25- or 23-gauge chandelier light. If not already present, a PVD up to the vitreous base was created intraoperatively, using a vitreous cutter.

\section{Results}

Five eyes of five patients were reviewed in this study. The characteristics of the eyes are shown in Table 1. The mean patient age was 20.4 ( \pm 9.47 standard deviation) years, ranging 13-35 years. The mean followup period was 36.4 ( $\pm 18.2 \mathrm{SD}$ ) months (range: 5-51 months). All patients were struck in the eye with a baseball at a relatively high speed, causing traumatic RD in two cases, traumatic $\mathrm{MH}$ in two cases, and RD due to traumatic $\mathrm{MH}$ in one case. Intraoperative findings at the beginning of vitrectomy showed that PVDs were not present in three cases and present in two cases. TA was used intraoperatively in all cases to facilitate the visualization of the vitreous. That enabled us to confirm, despite the PVD, the existence of the thin layer of localized vitreous adhesion to the retinal necrotic area, with/without retinal hole (not a horseshoe tear). The vitreous adhesion areas were localized to the superior quadrant in one case, the inferior quadrant in two cases, and the inferior temporal quadrant in two cases. All of the areas of localized vitreous adhesion were found between the major vascular arcades and the equator. The thin layer of the vitreous was removed with a vitreous cutter and diamond-dusted membrane scraper, but complete removal was impossible.

\section{Case I}

A 25-year-old male was struck in the right eye with a baseball (Figure 1A); he consulted an ophthalmologist, due to blurred vision in that eye. He was referred to our hospital for surgery for traumatic MH six weeks after the injury, because the MH had gradually expanded. The inferior retina showed widespread retinal hyperpigmentation, probably due to retinal necrosis. A PVD was created intraoperatively, using a vitreous cutter. TA was used to facilitate visualization. A thin layer of localized vitreous adhesion was observed at the retinal hyperpigmentation area, where it was not possible to remove all the vitreous adhesion. Some iatrogenic retinal breaks occurred intraoperatively, while removing the thin layer of residual vitreous cortex. The ILM was peeled off after the ICG staining procedure. After the fluid-air exchange, $1.2 \mathrm{~mL}$ of pure sulfur hexafluoride $\left(\mathrm{SF}_{6}\right)$ was injected into the vitreous cavity. MH closure was never obtained postoperatively, and two additional surgeries were necessary for pucker removal.

\section{Case 2}

A 35-year-old male was struck in the right eye with a baseball (Figure 1B). RD was found six weeks after the injury, and the patient was referred to our hospital for surgery. A PVD was 


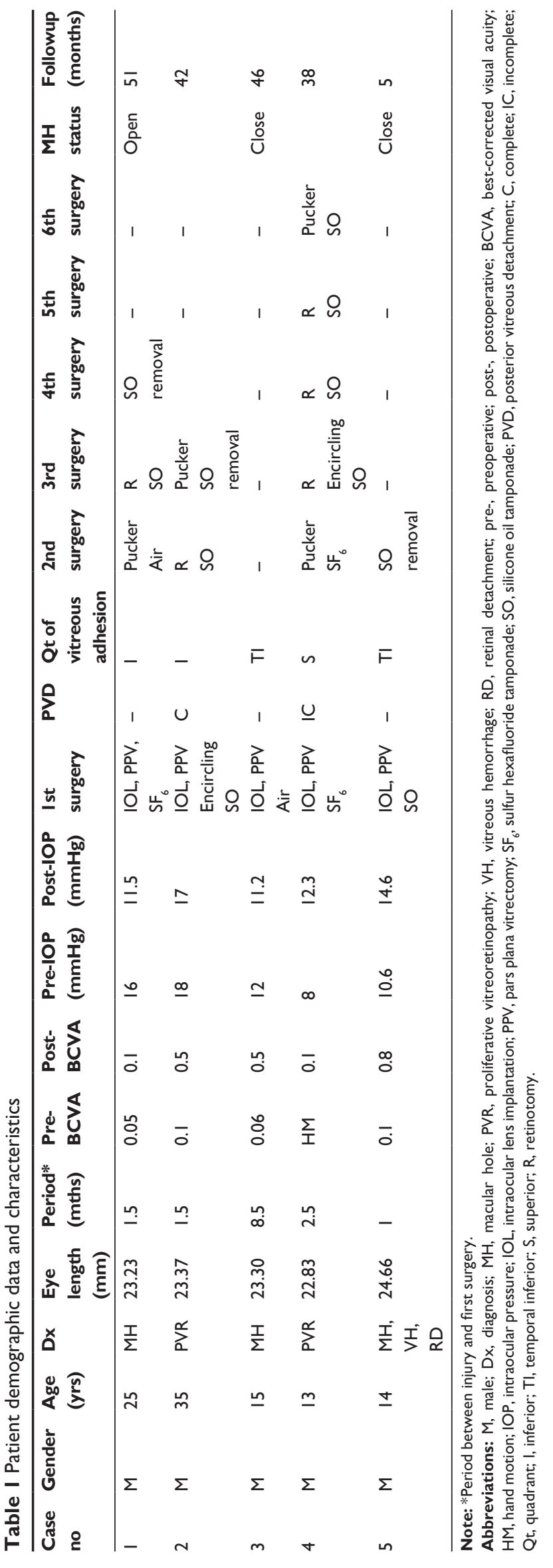

already present at the time of surgery. The inferior quadrant showed the retinal necrotic area, with many retinal breaks and subretinal proliferation. With the use of TA, vitreous adhesion was found overlapping the same area with the retinal necrosis, and complete removal of vitreous around that area was not possible. Some iatrogenic retinal breaks occurred intraoperatively while removing the thin layer of residual vitreous cortex. A combined encircling scleral buckle ( $3 \mathrm{~mm}$-wide silicone band) was placed at the equator, and SO was placed in the eye as an ocular tamponade. Initial retinal reattachment was not achieved in the early postoperative days. The stiffness of the inferior retina was considered to be the cause of $\mathrm{RD}$, and extensive retinotomy of the inferior quadrant was performed three days after the initial surgery. The additional surgery for pucker removal and SO removal was performed, and retinal reattachment was achieved.

\section{Case 3}

A 15-year-old boy was struck in the right eye with a baseball (Figure 1C). He was referred to our hospital eight and a half months after the injury for surgery for traumatic $\mathrm{MH}$. The inferior retina showed retinal hyperpigmentation, probably due to retinal contusion. A PVD was created intraoperatively, using a vitreous cutter. TA was used to facilitate visualization, and the localized vitreous adhesion was observed overlapping the retinal hyperpigmentation area. It was not possible to remove all of the vitreous adhesion. The ILM was peeled off, using TA. Fluid-air exchange was performed, and MH closure was achieved postoperatively.

\section{Case 4}

A 13-year-old boy was struck in the left eye with a baseball (Figure 1D). He was referred to our hospital ten weeks after the injury for surgery for RD in the superior quadrant. Intraoperatively, incomplete PVD was found, not extending up to the vitreous base. The surgical PVD was easily created up to the vitreous base, except in the superior quadrant, which showed a retinal necrotic area, with retinal breaks. With the use of TA, the localized vitreous adhesion was observed at the retinal necrotic area; complete vitreous removal was not possible. Some iatrogenic retinal breaks occurred intraoperatively, while removing the thin layer of residual vitreous cortex. After the fluid-air exchange, $0.5 \mathrm{~mL}$ of $\mathrm{SF}_{6}$ was injected. The patient required five additional surgeries due to proliferation, and retinal reattachment was obtained, with SO remaining in the eye. 


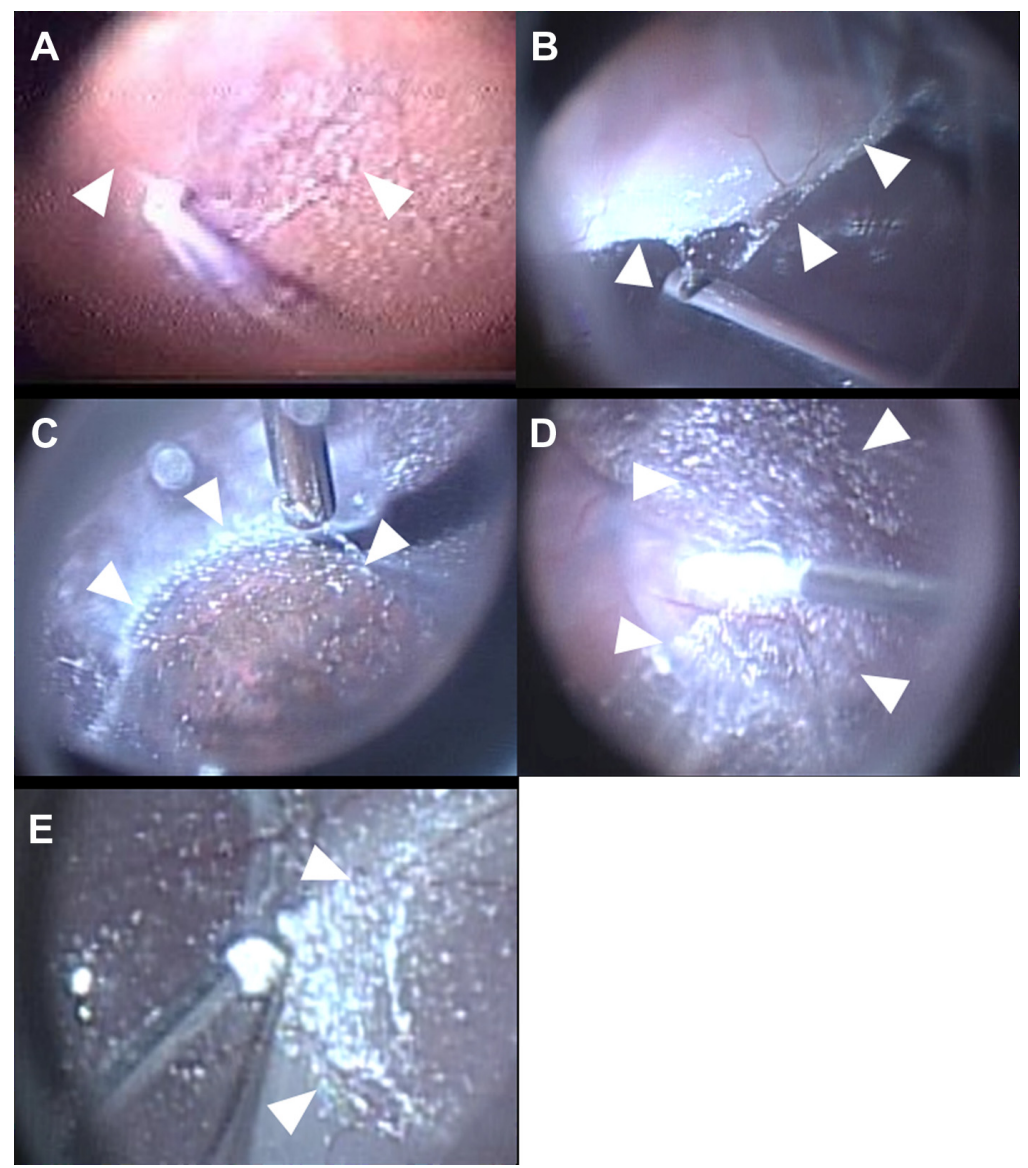

Figure I Intraoperative views of ocular contusion with a baseball. In all cases, a thin layer of localized vitreous adhesion (white arrowheads) was observed, overlapping the retinal degenerative areas, even after surgical posterior vitreous detachment. Triamcinolone acetonide was used to facilitate visualization of the vitreous and posterior hyaloid. (A) Case I, (B) Case 2, (C) Case 3, (D) Case 4, (E) Case 5.

\section{Case 5}

A 14-year-old boy was struck in the right eye with a baseball (Figure 1E), and he consulted an ophthalmologist for blurred vision. The fundus could not be visualized, due to vitreous hemorrhage. After a few days, traumatic $\mathrm{MH}$ and choroidal rupture were found. RD caused by MH was found later, and he was referred to our hospital four weeks after the injury. A PVD was created intraoperatively with a vitreous cutter. Localized vitreous adhesion was observed overlapping the retinal hyperpigmentation area; its complete removal was impossible. Some unintentional iatrogenic retinal breaks occurred intraoperatively. After the ICG staining procedure, the ILM was peeled, and the fluid-air exchange and SO injection were performed. The $\mathrm{SO}$ was removed eight weeks after the initial surgery. MH closure and retinal reattachment were achieved postoperatively.

\section{Discussion}

In our study, a thin layer of localized vitreous adhesion to the damaged retina was observed with the help of TA.
Visualization of the vitreous body during vitreoretinal surgeries was first introduced by Peyman and Sakamoto. ${ }^{11,18}$ Diffuse residual vitreous cortex after surgical PVD was reported to be much more frequent in diabetic retinopathy than in other diseases, ${ }^{11-14}$ and TA was essential to observe it. On the other hand, an "island-like" residual vitreous cortex, termed premacular residual vitreous cortex, frequently was found to be left on the macula after PVD in non-diabetic eyes. ${ }^{12,13,20}$ The premacular residual vitreous cortex can provide a good scaffold for cell proliferation and extracellular matrix production, resulting in future macular pucker and epiretinal membranes. ${ }^{21}$ Complete removal of the vitreous cortex, with the help of TA, has been shown to reduce the incidence of postoperative preretinal fibrous membrane formation post-vitrectomy. ${ }^{22}$

Cox divided retinal breaks by ocular contusion into the following three groups: Group 1- retinal breaks at the borders of vitreous; Group 2 - retinal holes in areas with no apparent vitreoretinal attachment; Group 3 - retinal tears that developed at the site of abnormal vitreoretinal attachment. ${ }^{23}$ 
Groups 1 and 3 may be the result of traction by the vitreous, due to deformation of the globe. ${ }^{8,9}$ On the other hand, Group 2 may be a consequence of direct concussional damage to the retina; it is unclear how the vitreous body plays a role in the development of this type of retinal break. Johnston also reported that $22.1 \%$ of patients who developed retinal breaks following an episode of ocular contusion had irregular breaks arising within the necrotic retina at the site of scleral impact, which was usually the inferior temporal quadrant. ${ }^{24}$ Cases 2 and 4 in our study showed retinal breaks in the retinal necrotic area; these two cases would belong to Cox's Group 2.

In our study, three cases (Cases 1, 2, and 4) required additional vitrectomies, due to postoperative proliferation. The residual vitreous cortex might have provided a scaffold for cell proliferation and tangential traction. Residual vitreous cortex around retinal breaks may especially result in proliferative changes. Case 1 did not show retinal tears, other than the macular hole at the preoperative examination. Some unintentional iatrogenic retinal breaks occurred intraoperatively after the meticulous vitreous removal, and additional vitrectomies were required, due to postoperative proliferation. To avoid iatrogenic retinal breaks, complete vitreous removal may not be necessary, unless there is residual vitreous cortex around the tears.

The mechanism of how localized vitreous adhesion develops is still unclear. One hypothesis is that unbalanced deformation between the globe and the vitreous body creates avulsion in the vitreous fibrae, and subsequently, the thin vitreous membrane is left on the retina. This also may be a secondary change in the vitreous body adjoining the damaged retina after ocular contusion. The vitreous has been reported to have a different viscosity and inertia than the surrounding ocular coats, leading to differential distortion and distension of the ocular contents during impact. ${ }^{8,9}$ Gregor et al reported structural change in the vitreous body following ocular contusion, using an experimental pig eye model. ${ }^{25}$ Four months after the injury, the eyes were enucleated for gross examination. The vitreous appeared normal and was attached to the posterior retina. ${ }^{25}$ In another report, the injured eyes underwent vitrectomies at ten weeks, and microscopic examination revealed that a thin layer of vitreous cortex lined the entire retina in eight of ten eyes..$^{10}$ These studies may suggest the existence of structural changes in the vitreous body in injured eyes, and that the connection between the thin vitreous membrane on the retina and the core vitreous may differ from those in non-injured eyes. However, this may not totally support our findings of the localized vitreous adhesion overlapping damaged retinal areas, as their findings were a thin layer of vitreous cortex lining the entire retina.

In conclusion, a thin layer of localized, vitreous adhesion at the area of retinal degeneration was observed in eyes following ocular contusion. With the existence of this thin layer in mind, TA may be useful during vitrectomies. Further studies are needed to clarify the mechanism of localized vitreous adhesion, and to determine whether complete removal of the vitreous is necessary.

\section{Disclosure}

The authors report no conflicts of interest in this work.

\section{References}

1. Kohno T, Ishibashi T, Inomata H, Ikui H, Taniguchi Y. Experimental macular edema of commotio retinae: preliminary report. Jpn J Ophthalmol. 1983;27(1):149-156.

2. Hui YN, Wu YQ, Xiao QS, Kirchhof B, Heimann K. Repair of outer blood-retinal barrier after severe ocular blunt trauma in rabbits. Graefes Arch Clin Exp Ophthalmol. 1993;231(6):365-369.

3. Hart JC, Frank HJ. Retinal opacification after blunt non-perforating concussional injuries to the globe. A clinical and retinal fluorescein angiographic study. Trans Ophthalmol Soc UK. 1975;95(1):94-100.

4. François J, De Laey JJ. Fluoroangiographic study of traumatic chorioretinal angiopathy. Bull Soc Belge Ophtalmol. 1970;156:577-589.

5. COGAN DG. Primary chorioretinal aberrations with night blindness; pathology. Trans Am Acad Ophthalmol Otolaryngol. 1950;54: 629-661.

6. Crouch ER Jr, Apple DJ. Posttraumatic migration of retinal pigment epithelial melanin. Am J Ophthalmol. 1974;78(2):251-254.

7. Bastek JV, Foos RY, Heckenlively J. Traumatic pigmentary retinopathy. Am J Ophthalmol. 1981;92(5):621-624.

8. Weidenthal DT, Schepens CL. Peripheral fundus changes associated with ocular contusion. Am J Ophthalmol. 1966;62(3):465-477.

9. Delori F, Pomerantzeff O, Cox MS. Deformation of the globe under high-speed impact: its relation to contusion injuries. Invest Ophthalmol. 1969;8(3):290-301.

10. Gregor Z, Ryan SJ. Combined posterior contusion and penetrating injury in the pig eye. III. A controlled treatment trial of vitrectomy. $\mathrm{Br}$ J Ophthalmol. 1983;67(5):282-285.

11. Sakamoto T, Miyazaki M, Hisatomi T, et al. Triamcinolone-assisted pars plana vitrectomy improves the surgical procedures and decreases the postoperative blood-ocular barrier breakdown. Graefes Arch Clin Exp Ophthalmol. 2002;240(6):423-429.

12. Doi N, Uemura A, Nakao K, Sakamoto T. Vitreomacular adhesion and the defect in posterior vitreous cortex visualized by triamcinoloneassisted vitrectomy. Retina. 2005;25(6):742-745.

13. Sonoda KH, Sakamoto T, Enaida H, et al. Residual vitreous cortex after surgical posterior vitreous separation visualized by intravitreous triamcinolone acetonide. Ophthalmology. 2004;111(2):226-230.

14. Matsumoto H, Yamanaka I, Hisatomi T, et al. Triamcinolone acetonideassisted pars plana vitrectomy improves residual posterior vitreous hyaloid removal: ultrastructural analysis of the inner limiting membrane. Retina. 2007;27(2):174-179.

15. Ikuno Y, Sayanagi K, Ohji M, et al. Vitrectomy and internal limiting membrane peeling for myopic foveoschisis. Am J Ophthalmol. 2004;137(4):719-724.

16. Le Rouic JF, Ducournau D, Becquet F. Triamcinolone-assisted vitrectomy in the treatment of recurrent retinal detachment due to myopic macular hole. J Fr Ophtalmol. 2006;29(7):829-834. 
17. Yamamoto N, Ozaki N, Murakami K. Triamcinolone acetonide facilitates removal of the epiretinal membrane and separation of the residual vitreous cortex in highly myopic eyes with retinal detachment due to a macular hole. Ophthalmologica. 2004;218(4):248-256.

18. Peyman GA, Cheema R, Conway MD, Fang T. Triamcinolone acetonide as an aid to visualization of the vitreous and the posterior hyaloid during pars plana vitrectomy. Retina. 2000;20(5):554-555.

19. Nishimura A, Kobayashi A, Segawa Y, et al. Isolating triamcinolone acetonide particles for intravitreal use with a porous membrane filter. Retina. 2003;23(6):777-779.

20. Yamashita T, Sakamoto T, Yamakiri K, et al. Polylactic acid for visualizing the vitreous body during vitrectomy. Invest Ophthalmol Vis Sci. 2007;48(7):3277-3282.
21. Chen TY, Yang CM, Liu KR. Intravitreal triamcinolone staining observation of residual undetached cortical vitreous after posterior vitreous detachment. Eye (Lond). 2006;20(4):423-427.

22. Enaida H, Hata Y, Ueno A, et al. Possible benefits of triamcinoloneassisted pars plana vitrectomy for retinal diseases. Retina. 2003;23(6): 764-770.

23. Cox MS, Schepens CL, Freeman HM. Retinal detachment due to ocular contusion. Arch Ophthalmol. 1966;76(5):678-685.

24. Johnston PB. Traumatic retinal detachment. Br J Ophthalmol. 1991; 75(1):18-21.

25. Gregor Z, Ryan SJ. Combined posterior contusion and penetrating injury in the pig eye. II. Histological features. $\mathrm{Br} J$ Ophthalmol. 1982;66(12):799-804.
Clinical Ophthalmology

\section{Publish your work in this journal}

Clinical Ophthalmology is an international, peer-reviewed journal covering all subspecialties within ophthalmology. Key topics include: Optometry; Visual science; Pharmacology and drug therapy in eye diseases; Basic Sciences; Primary and Secondary eye care; Patient Safety and Quality of Care Improvements. This journal is indexed on

\section{Dovepress}

PubMed Central and CAS, and is the official journal of The Society of Clinical Ophthalmology (SCO). The manuscript management system is completely online and includes a very quick and fair peer-review system, which is all easy to use. Visit http://www.dovepress.com/ testimonials.php to read real quotes from published authors. 